\title{
A Plug and Play Module to identify type of Modulation
}

\author{
Varadaraju H.R ${ }^{1}$, Dr.Senthil Kumar.K ${ }^{2}$ andDr. Ravi. $S^{3}$ \\ ${ }^{1}$ Research Scholar and $d^{2,3}$ Professor \\ Department of Electronics and Communication Engineering \\ Dr. MGR Educational and Research Institute University, Chennai, India \\ Email: hrvrsiet@gmail.com
}

\begin{abstract}
The main goal of this work is to identify the type of modulation in an independent manner with the use of plug and play module. As, some of the applications of SDR involve military and relief operation (non-cooperative relay environments), wireless data is sent using a suitable modulation technique to provide suboptimal and optimal receivers, savings in power for a required bit error rate etc. The constraints like limited available frequency bandwidth, time-varying multipath fading, shadowing and mobility are considered in this work.Efficient techniques to overcome these limitations involve adaptively changing the modulation mode based on the channel quality information sensed by the receiver and fed back to the transmitter to the throttling of the window size by the sender. The receiver should identify the type of modulation the transmitter has sent
\end{abstract}

Keywords: DPSK, Adaptive modulation

\section{Introduction}

A wireless channel model can be considered both as a time-domain and frequency-domain channel with nonstationary and ergodic features. As a time-domain channel it is complex normal and independent across filter taps and users. Similarly in frequency-domain it has correlated channel gains across subcarriers. The general multidimensional linear channel model adequately represents a plethora of communication system models which utilize multidimensional transmit-receive signals for attaining increased rates and reliability in the presence of fading. Proposed method used to enhance feature extraction schemes, to optimize the number of feature vectors considered for identification and to combine deterministic and blind autonomous modulation identification schemes. The fundamental feature vectors are needed to be identified and used.

\section{Related works}

HadiValipour, $\mathrm{M}$ et al., [2012] have proposed a new method for automatic digital modulation classification in presence of AWGN. In this method a set of three different types of features is extracted to be employed in recognition process. Classification is based on SVM as a powerful method for pattern recognition, and PSO to configure kernel parameters [1].

By referring to the paper by Jaspal Bagga \& Neeta Tripathi [2011] in which the results of experiment carried out on a digitally modulated signals concludes that analysis using instantaneous and random features classification, we understood that: A receiver with completely non informative about the modulation scheme has inferior ability than a receiver used in a wireless Network with ability to detect the autonomous modulation scheme for detection. [2].

However based on the signal and Noise ratio (SNR) classification of modulated signals can be done in which noise interference is used as factor to classify the modulation scheme and is demonstrated in the paper published by Tao He \&Zheng-Ou Zhou in 2008 [3].

Further the classification of M-array frequency shift keying signals can be done based on the analysis of complex envelope particularly the analysis of cyclostationary statistics modulated signals their periodicity was proved to be the better way to classify the modulated signal which was proposed and presented by AnttiVeikkoRosti\& Visa Koivunen in 2000[4].

Prakasam, P \&Madheswaran, M [2008] presented that by adopting the wavelet transform and statistical parameters the digital modulation identification model can be improved further. In this method the dynamic methodology was effectively used with the help of a priori unknown paprametersto identify the modulation schemes of M-ary PSK, M-ary QAM, GMSK and M-ary FSK modulations [5].

Liang Hong published paper in 1999, which deals with automatic identification of digitally modulated signal through transients in amplitude, frequency or phase was considered as a rapidly evolving field in the application areas like electronic warfare, surveillance and threat analysis was presented in this paper. [6]. 


\section{Adaptive modulation}

The identification of modulation scheme can be done through either [12] Decision Theoretic approach in which likelihood functions were used for probabilistic and hypothesis testing of arguments or Statistical Pattern approach[10][11] in which prominent characteristics of the signals are extracted and analysed to find the modulation scheme. Other ways of identification of modulation scheme is through i) Spectral Processing ii) Instantaneous amplitude, Phase and Frequency parameters iii) Instantaneous Amplitude, phase and frequency histogram iv) Combination of all the above three types and v) Universal demodulators. The Decision theoretic approach uses the likelihood function where arguments to formulate the recognition problem and classification is performed. The drawback with this approach is its high computational complexity, difficulty in implementation and lack of robustness to model mismatch. The pattern recognition approach is based on feature extraction and recognition in which the prominent characteristics of the signal is extracted and analysed to indicate the modulation type. The important metrics used in this type of analysis are interference and system capacity. In a radio communication system the major bottle neck is the interference which causes the cross talks on voice channels and may results in severe problem like call dropping in the channels.

Normally Interferences may occur due to any of the following reasons,

- A mobile station working in the same base cell

- There may be a ongoing call in the neighboring cell

- If any base station operates in the frequency range of currently operating cell

The interferences are of two types

i. Co-channel Interferences and

ii. Adjacent Channel Interferences.

\section{Co-channel Interference}

It can be interference between signals from co-channel nodes [8]. The preferred way to reduce this interference is to separate nodes by minimum distance ' $D$ ' in a cell radius ' $R$ ' which results in a Co-channel reuse ratio is given by

$$
\mathrm{Q}=\frac{\mathrm{D}}{\mathrm{R}}=\sqrt{3 \mathrm{~N}}
$$

Where, $\mathrm{N}$-cluster size for hexagonal geometry

where $\mathrm{i}_{0}=$ the number of Co-channel interfering nodes, the signal to interference ratio (SIR) is

$$
\frac{\mathrm{S}}{\mathrm{I}}=\frac{\mathrm{S}}{\sum_{\mathrm{i}=1}^{\mathrm{i}_{0} \mathrm{I}_{\mathrm{i}}}}
$$

In (2) $\mathrm{S}$ represents the desired signal power from its base station and

$\mathrm{I}_{\mathrm{i}}$ is the interference power caused by $\mathrm{i}^{\text {th }}$ interference co channel station.

The average received power $\mathrm{P}_{\mathrm{r}}$ at a distance ' $\mathrm{d}$ ' from the transmitting antenna as per the poynting vector theorem

Hers $P_{o}=$ received power

$$
P_{r}=P_{0}\left(d / d_{0}\right)^{-n}
$$

$\mathrm{d}_{\mathrm{o}}=\mathrm{s}$ the distance from the transmitting antenna

$\mathrm{n}=$ path loss exponent (ranges between 2 and 4 )

Equation (3) can also be written as,

$$
P_{r}(d B m)=P_{0}(d B m)-10 \log \left(d / d_{0}\right)
$$

If all the interference base stations are equidistant from the desired base station, the signal to noise interference ratio is given by

$$
\frac{S}{R}=\frac{(D / R)^{n}}{i_{0}}=\frac{(\sqrt{3 N})^{n}}{i_{0}}
$$

Channel support for Time slot based modulation schemes (TSMA)

Number of channels in Time slot based modulation schemes system is given by

$$
\mathrm{N}=\frac{\mathrm{m}\left(\mathrm{B}_{\text {tot }}-2 \mathrm{~B}_{\text {guard }}\right)}{\mathrm{B}_{\mathrm{c}}}
$$

Where $\mathrm{m}$ represents the maximum number of TSMA users on radio channel, 2Bguard (one frequency band allocated at the low end and other at the high end) and $B_{c}$ is the channel bandwidth.

\section{Capacity of Users}

Let the number of users be $\mathrm{N}$. The signal to noise ratio is given by

$$
\mathrm{SNR}=\frac{\mathrm{S}}{(\mathrm{N}-1) \mathrm{S}}=\frac{1}{\mathrm{~N}-1}
$$

Where, $\mathrm{S}$ is the desired signal power and $\mathrm{N}-1$ is the number of interfering users

The signal to noise ratio at the base station receiver can be represented in terms E0/N0.

$$
\mathrm{E}_{0} / \mathrm{N}_{0}=\frac{\mathrm{S} / \mathrm{R}}{(\mathrm{N}-1)(\mathrm{S} / \mathrm{W})}=\frac{\mathrm{W} / \mathrm{R}}{\mathrm{N}-1}
$$


Where, $\mathrm{S}$ is the signal power and $\mathrm{R}$ is the base band information bit rate. Bit energy to noise is obtained by dividing signal power by base band information bit rate $\mathrm{R}$.

\section{Automatic Digital Modulation Recognition in Presence of Noise}

Automatic digital modulation[13] recognition is implemented in the presence of AWGN. This method employs three different types of features which are extracted to perform the recognition process[7]. They are Spectral based features, Statistical features and Wavelet based features. Hierarchical structure for classification of signals[16] is shown in Figure1. The first layer in the structure detects the incoming modulation type as either ASK, FSK, PSK or QAM. The next layer implements the corresponding classifiers to identify the modulation type's order (for example, 2ASK, 4ASK, 8ASK or 16ASK).

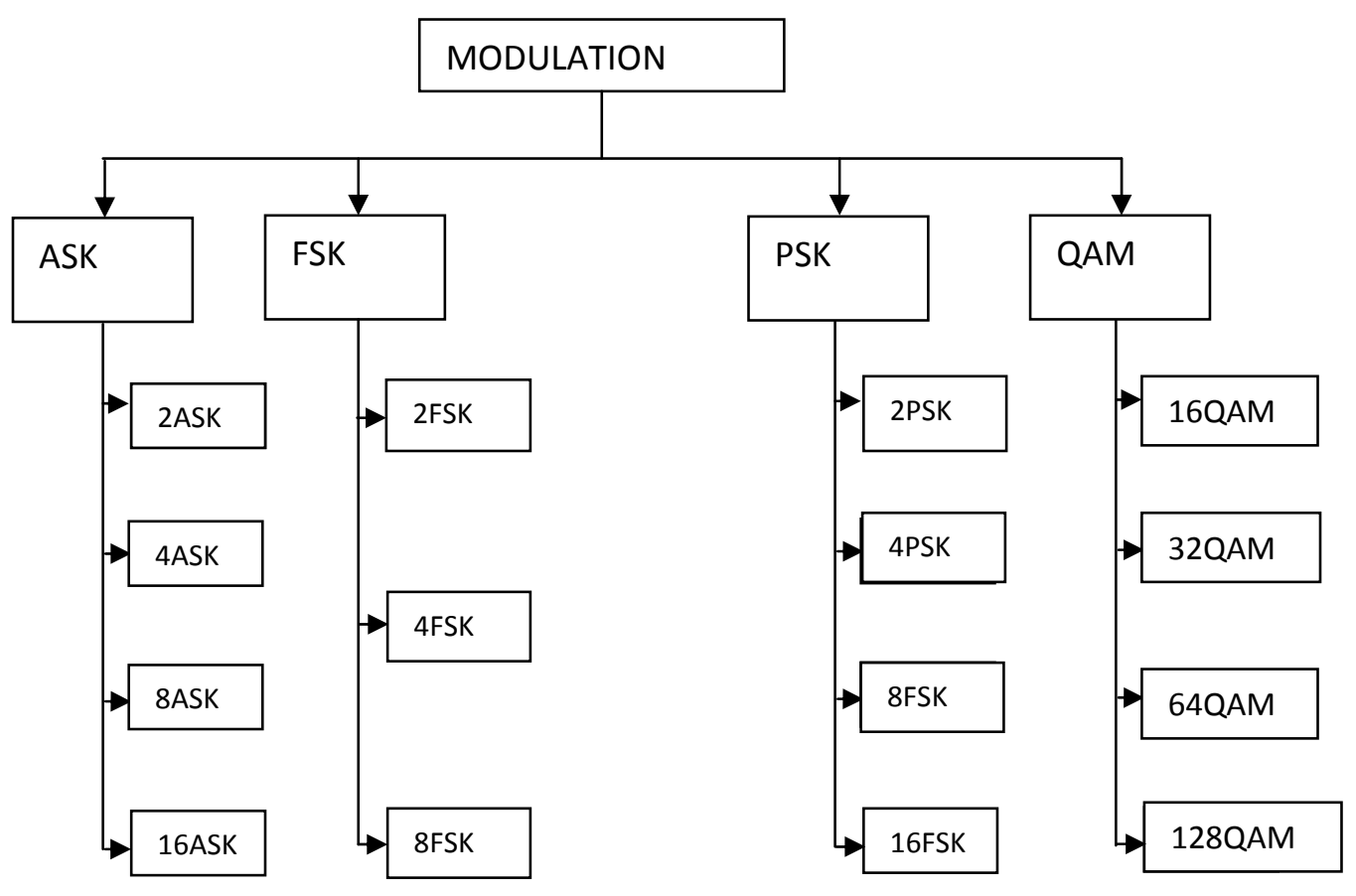

Figure 1: Modulation types using Hierarchical structure

Different types of digitally modulated signals (corrupted by AWGN) are simulated to measure the performance and suitability of the method. The following Table 1 shows the features and expressions for various types of modulations[14].

Table1. Various types of modulations with their features

\begin{tabular}{|l|c|l|}
\hline Signal & Expression & Features \\
\hline \multicolumn{2}{|c|}{ Amplitude Modulation } \\
\hline Amplitude modulation & $\mathrm{A}[1+\mathrm{ms}(\mathrm{t})] \cos \omega_{\mathrm{c}} \mathrm{t}$ & $\begin{array}{l}\text { Modulation and demodulation is simple } \\
\text { Bandwidth of } \mathrm{S}(\mathrm{t}) \text { is B Hertz }\end{array}$ \\
\hline $\begin{array}{l}\text { Double } \\
\text { modulation }\end{array}$ & $\mathrm{s}(\mathrm{t}) \cos \omega_{\mathrm{c}} \mathrm{t}$ & $\begin{array}{l}\text { Modulation simple but demodulation is } \\
\text { difficult. Power requirement is } 50 \% \text { less than } \\
\text { that of AM. }\end{array}$ \\
\hline $\begin{array}{l}\text { Quadrature amplitude } \\
\text { modulation }\end{array}$ & $\mathrm{s}_{1}(\mathrm{t}) \cos \omega_{\mathrm{c}} \mathrm{t}+\mathrm{s}_{2}(\mathrm{t}) \sin \omega_{\mathrm{c}} \mathrm{t}$ & Bandwidth requirement is less \\
\hline $\begin{array}{l}\text { Single } \\
\text { modulation }\end{array}$ & $\frac{1}{2}\left[\mathrm{~s}_{1}(\mathrm{t}) \cos \omega_{\mathrm{c}} \mathrm{t}+\mathrm{s}_{2}(\mathrm{t}) \sin \omega_{\mathrm{c}} \mathrm{t}\right]$ & $\begin{array}{l}\text { Bandwidth requirement is less but difficult to } \\
\text { demodulate }\end{array}$ \\
\hline $\begin{array}{l}\text { Vestigial sideband } \\
\text { modulation }\end{array}$ & $\frac{1}{2}\left[\mathrm{~s}(\mathrm{t}) \cos \omega_{\mathrm{c}} \mathrm{t}+\mathrm{js}(\mathrm{t}) \sin \omega_{\mathrm{c}} \mathrm{t}\right]$ & $\begin{array}{l}\text { Bandwidth requirement is less but difficult to } \\
\text { demodulate. Only a half the sideband is used. }\end{array}$ \\
\hline \multicolumn{2}{|c|}{$\mathrm{A} \cos \left[\omega_{\mathrm{c}} \mathrm{t}+\mathrm{s}(\mathrm{t})\right.$} & $\begin{array}{l}\text { Difficult to implement. Has distortion and } \\
\text { noise durability. }\end{array}$ \\
\hline Phase modulation & Acos $\left[\omega_{\mathrm{c}} \mathrm{t}+\int \mathrm{s}(\mathrm{t}) \mathrm{dt}\right.$ & $\begin{array}{l}\text { Modulation and demodulation simple. Has } \\
\text { distortion and noise durability. }\end{array}$ \\
\hline Frequency modulation & \multicolumn{2}{|c}{}
\end{tabular}




\begin{tabular}{|c|c|c|}
\hline \multicolumn{3}{|c|}{ Pulse Modulation } \\
\hline $\begin{array}{ll}\text { Pulse } & \text { amplitude } \\
\text { modulation } & \end{array}$ & $\sum^{\infty}[\operatorname{as}(n T)+K] \pi(\mathrm{t}-\mathrm{nT} / \tau)$ & $\begin{array}{l}\text { Modulation and demodulation simple. Has } \\
\text { distortion and noise durability. }\end{array}$ \\
\hline $\begin{array}{ll}\text { Pulse } & \text { duration } \\
\text { modulation } & \end{array}$ & $\begin{array}{l}\sum_{\mathrm{n}=\infty}^{\infty} \pi(\mathrm{t}-\mathrm{nT} / \tau(\mathrm{s})) \\
\tau(\mathrm{s})=\operatorname{as}(\mathrm{nT})+\mathrm{K}\end{array}$ & $\begin{array}{l}\text { Amplitude is not affected by noise but sides of } \\
\text { pulse are affected by noise. }\end{array}$ \\
\hline $\begin{array}{l}\text { Pulse } \\
\text { modulation }\end{array}$ & $\begin{array}{l}\sum_{\mathrm{n}=\infty}^{\infty} \pi(\mathrm{t}-\mathrm{nT}-\alpha / \tau(\mathrm{s})) \\
\alpha=\operatorname{as}(\mathrm{nT})\end{array}$ & $\begin{array}{l}\text { Pulses are equal height and width. Only one } \\
\text { side of the pulse affected by noise. }\end{array}$ \\
\hline \multicolumn{3}{|c|}{ Digitized form of Analog signal } \\
\hline Pulse code modulation & $\begin{array}{l}\mathrm{BW}=2 \mathrm{nB}, \quad \mathrm{N}=\mathrm{No} . \\
\text { quantization, } \mathrm{B}=\mathrm{BW} \text { of the } \\
\text { analog signal }\end{array}$ & $\begin{array}{l}\text { Modulation and demodulation simple so } \\
\text { widely used. Sturdy against noise and } \\
\text { distortion hence used for high quality } \\
\text { transmission. }\end{array}$ \\
\hline \multicolumn{3}{|c|}{ Binary Modulation } \\
\hline Amplitude shift keying & $h(t) \cos \omega_{c} t, \quad h(t)=\left\{\begin{array}{l}0 \\
1\end{array}\right.$ & $\begin{array}{l}\text { Modulation and demodulation simple. Sturdy } \\
\text { against noise and distortion. }\end{array}$ \\
\hline Frequency shift keying & $\cos \left[\omega_{h}(t) t\right], \quad \omega_{h}(t)=\left\{\begin{array}{l}2 \pi f_{0} \\
2 \pi f_{1}\end{array}\right.$ & $\begin{array}{l}\text { High energy requirement than ASK. } \\
\text { Implementation complicated. Sturdy against } \\
\text { noise and distortion. }\end{array}$ \\
\hline Phase shift keying & $\cos [\omega(\mathrm{t})+\emptyset(\mathrm{t})], \quad \phi(\mathrm{t})=\left\{\begin{array}{l}0 \\
\pi\end{array}\right.$ & $\begin{array}{l}\text { High energy requirement than ASK. } \\
\text { Implementation complicated. }\end{array}$ \\
\hline
\end{tabular}

\section{DPSK modulation and Detection}

In DPSK modulation, in step1 data is differentially encoded by using comparator/decision block. In step 2, the differently encoded data is phase encoded. The block diagram of DPSK transmitter and the DPSK pulse stream with noise are shown in figure 2 and figure 3.

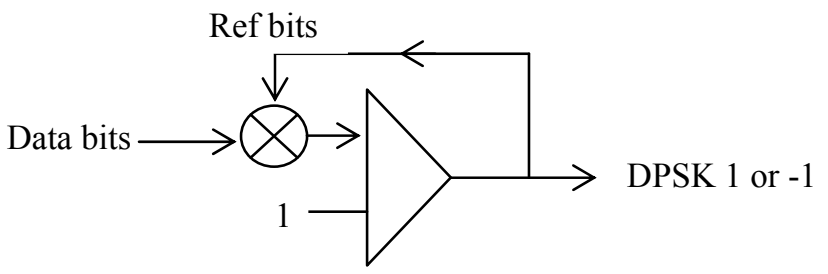

Figure 2 DPSK transmitter

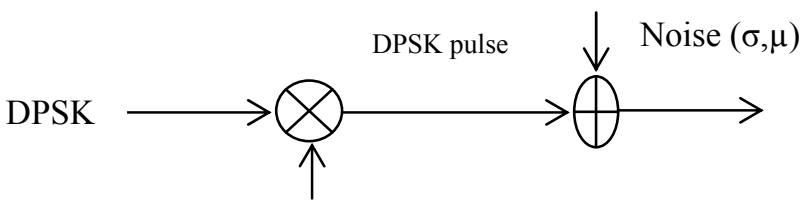

$\operatorname{Cos} 2 \pi f_{c} t$

Figure 3 DPSK pulse stream with noise

\section{DPSK bit generation}

The table 2. Below is DPSK bit generation 
Table 2. DPSK bit generation

\begin{tabular}{|c|c|c|c|c|}
\hline $\mathrm{k}_{\mathrm{f}}$ & $\mathrm{a}\left(\mathrm{k}_{\mathrm{f}}-1\right)$ & $\mathrm{ad}\left(\mathrm{k}_{\mathrm{f}}-1\right) \mathrm{ad}[1]=1$ & $\mathrm{a}_{\mathrm{r}}$ & $\mathrm{a}_{\mathrm{d}}\left(\mathrm{k}_{\mathrm{f}}\right)$ \\
\hline 2 & $\mathrm{a}[1]=1$ & $\mathrm{ad}[2]=1$ & 1 & 1 \\
\hline 3 & $\mathrm{a}[2]=1$ & $\mathrm{ad}[3]=1$ & 1 & 1 \\
\hline 4 & $\mathrm{a}[3]=-1$ & $\mathrm{ad}[4]=-1$ & -1 & -1 \\
\hline 5 & $\mathrm{a}[4]=-1$ & $\mathrm{ad}[5]=1$ & 1 & 1 \\
\hline 6 & $\mathrm{a}[5]=-1$ & $\mathrm{ad}[6]=-1$ & -1 & -1 \\
\hline 7 & $\mathrm{a}[6]=1$ & $\mathrm{ad}[7]=-1$ & -1 & -1 \\
\hline 8 & $\mathrm{a}[7]=-1$ & $\mathrm{ad}[8]=1$ & 1 & 1 \\
\hline 9 & $\mathrm{a}[8]=1$ & $\operatorname{ad}[9]=1$ & 1 & 1 \\
\hline
\end{tabular}

The Matlab tool is used to analysis the performance of DPSK transmitter. The pseudo code for DPSK bit generation is given by,

For $\mathrm{i}=0$ to $2: \mathrm{N}+1$

$\operatorname{ar}=\mathrm{a}(\mathrm{kf}-1)^{*} \mathrm{ad}(\mathrm{kf}-1)$

ifar equal to 1

$\operatorname{ad}(\mathrm{kf})=1$

else

$\operatorname{ad}(\mathrm{kf})=-1$

end if

\section{Simulated results}

The generated DPSK bits with 0.01 additive white noise is shown in figure 4 .

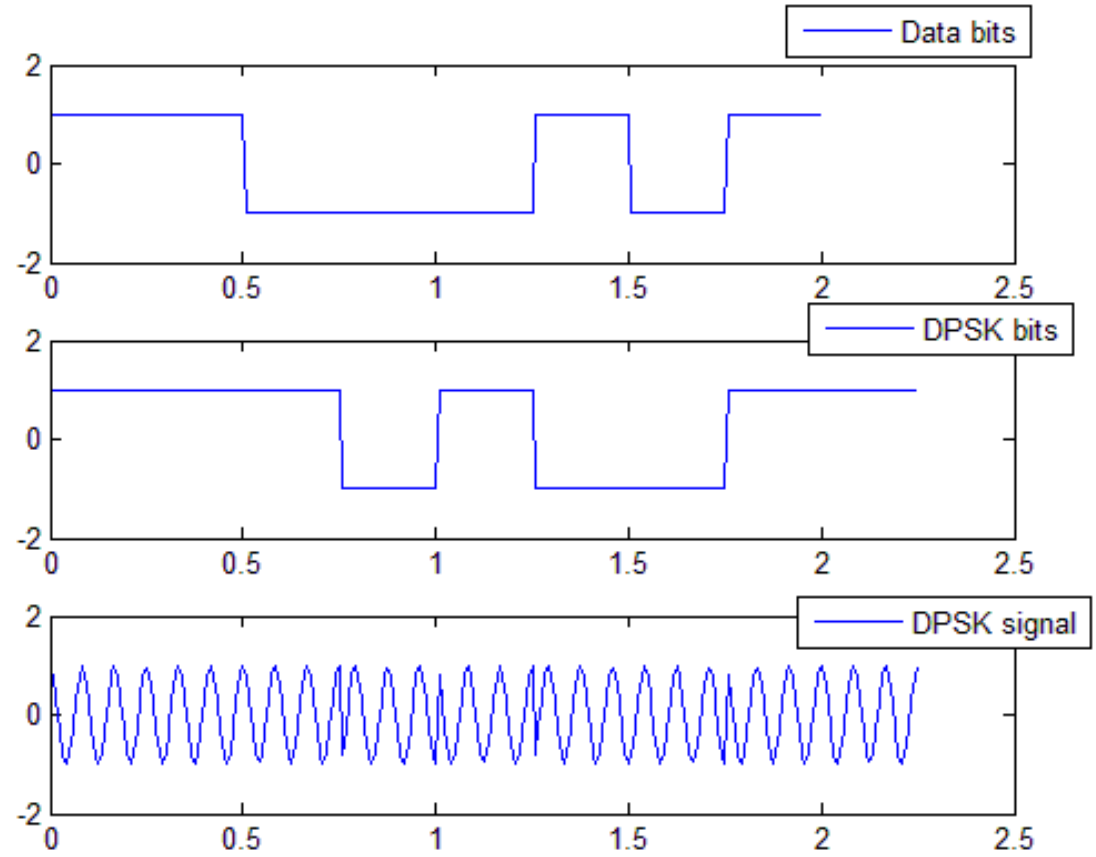

Figure 4 DPSK signal 


\section{Information/ entropy analysis from extracted features}

\section{Information Calculation}

Table 3 shows the relation between the features extracted from the samples or frames and the DPSK modulation signal. Features related to each sample[13] are checked and the amount of information is calculated[17].

Table 3 Features of sample for DPSK modulation signal

\begin{tabular}{|c|c|c|c|c|}
\hline Frame/sample & Feature F1 & Feature F2 & Feature F3 & DPSK signal \\
\hline 1 & Yes & Yes & 1 & Yes \\
\hline 2 & Yes & Yes & 6 & Yes \\
\hline 3 & Yes & No & 5 & No \\
\hline 4 & No & No & 4 & Yes \\
\hline 5 & No & Yes & 7 & No \\
\hline 6 & No & Yes & 3 & No \\
\hline 7 & No & No & 8 & No \\
\hline 8 & Yes & No & 7 & Yes \\
\hline 9 & No & Yes & 5 & No \\
\hline
\end{tabular}

Entropy for the DPSK signal from the above training samples can be calculated as

$\mathrm{p}(\mathrm{y})=4 / 9 ; \mathrm{p}(\mathrm{n})=5 / 9$;

$\mathrm{H}=-\mathrm{p}(\mathrm{y}) \log 2 \mathrm{p}(\mathrm{y})-\mathrm{p}(\mathrm{n}) \log 2 \mathrm{p}(\mathrm{n})=0.9911$

The information gains for the two features using Boolean type F1 and F2 for these samples are calculated as follows,

\section{For feature F1}

\begin{tabular}{|c|c|c|}
\hline Feature F1 & No. of times DPSK signal is Positive & No. of times DPSK signal is Negative \\
\hline Yes & $3[$ i.e. samples 1,2 and 8$]$ & $1[$ i.e. sample 3] \\
\hline No & $1[$ i.e. sample 4] & $4[$ i.e. samples 5,6,7,9] \\
\hline
\end{tabular}

Entropy for feature $\mathrm{F} 1$ is given as

$$
\begin{aligned}
\mathrm{H} \mathrm{fl} & =4 / 9[-3 / 4 \log 23 / 4-1 / 4 \log 21 / 4]+5 / 9[-1 / 5 \log 21 / 5-4 / 5 \log 24 / 5] \\
& =0.7616
\end{aligned}
$$

Information gain for feature F1=Total Entropy- feature F1 Entropy

$$
=0.9911-0.7617=0.2294
$$

\section{For feature F2}

\begin{tabular}{|c|c|c|}
\hline Feature F2 & No. of times DPSK signal is Positive & No. of time DPSK signal is Negative \\
\hline Yes & 2[i.e. samples 1,2$]$ & [ [i.e. samples $5,6,9]$ \\
\hline No & 2[i.e. sample 4,8] & 2[i.e. samples 3,7] \\
\hline
\end{tabular}

Entropy for $\mathrm{F} 2$ is given as

H f2 $=5 / 9[-2 / 5 \log 22 / 5-3 / 5 \log 23 / 5]+4 / 9[-2 / 4 \log 22 / 4-2 / 4 \log 22 / 4]=0.9839$

Information gain for feature F2=Total Entropy- feature F2 Entropy

$$
=0.9911-0.9839=0.0072
$$

\section{Performance metrics}

To achieve bit error rate of $10^{-5}$ in both DPSK and coherent BPSK, the excess power margin is required in DPSK is $0.75 \mathrm{~dB}$. Thus coherent BPSK requires lesser power. The required power margin for various bit error rate is shown in table 4.

Table 4 Power requirement to achieve bit error rate

\begin{tabular}{|c|c|}
\hline To achieve required BER & Excess power margin required in DPSK in dB \\
\hline $10^{-5}$ & 0.75 \\
\hline $10^{-4}$ & 0.9 \\
\hline $10^{-3}$ & 1.1 \\
\hline $10^{-2}$ & 1.6 \\
\hline
\end{tabular}


Thus, if a system can achieve higher BER, then excess power margin required in DPSK is reduced[19] and in such cases DPSK can be tolerated compared to Coherent BPSK. This is shown in figure 5.

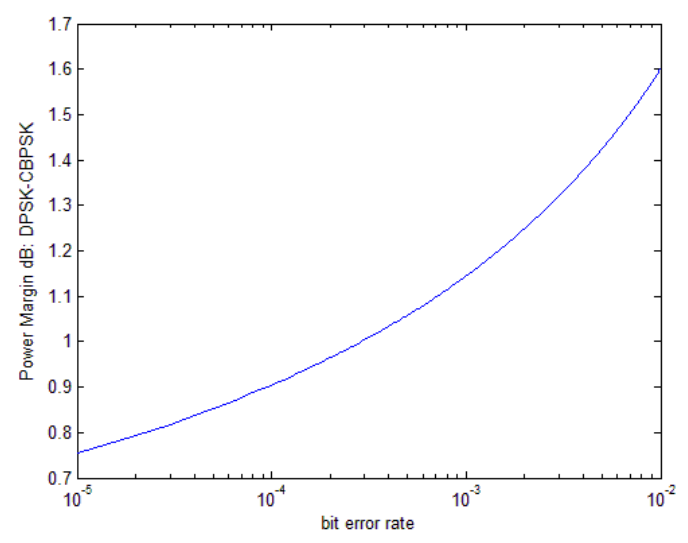

Figure 5 Power margin Vs Bit error rate

\section{Conclusion}

In this paper, anticipative models where decisions are made based on the distribution and the actual realizations of the random quantities (adaptive models) are presented for MIMO system. A unique premodulation filter along with an adaptive code synchronization scheme is studied. Multistage recourse models with nonanticipative action for the $1^{\text {st }}$ stage and recourse actions for the $2^{\text {nd }}$ stage based on the realization of the random quantities are presented.Channel estimation models and self-healing noise filter modules are included to make the system robust.The proposed work shall assist in improved definitive prioritization among users and can trace boundary of capacity region with specified ratio. The proposed algorithms are adaptive with low complexity and more suited for online processing.Hardware implementation using embedded open source tools (Linux kernel) and Reconfigurable hardware is the future scope of this work.

\section{References}

[1] HadiValipour, M, MediHomayounpour, M\& Amin Mehralian, M (eds) 2012, Proceedings of the $6^{\text {th }}$ International Symposium on Telecommunication, November 6-, 2012: Automatic Digital Modulation inPresence of Noise Using SVM and PSO. IEEE, Tehra

[2] JaspalBagga\& Neeta Tripathi 2011, 'Analysis of Digitally Modulated Signals using Instantaneous andStochastic Features, for Classification', International Journal of Soft Computing and Engineering, vol. 1, no. 2,pp. 57-61

[3] Tao He \&Zheng-Ou Zhou 2008, 'Classification of Modulated Signals using Multifractal Features', Journal ofthe Chinese Institute of Engineers, vol. 31 , no. 2, pp. 335-338

[4] Antti-VeikkoRosti\& Visa Koivunen 2000, Proceedings of the European Signal Processing Conference 2000,September 4-8, 2000: Classification of Mfsk Modulated Signals using the Mean of Complex Envelope. EuropeanAssociation of Signal Processing, Tampre, Finland

[5] Prakasam, P \&Madheswaran, M 2008, 'Digital Modulation Identification Model Using Wavelet Transform and Statistical Parameters', Journal of Computer Systems, Networks and Communications,

[6] Liang Hong 1999, Proceedings of the Military Communications Conference, October 31-November 3, 1999:Identification of Digital Modulation types using the Wavelet Transform. IEEE Soc., Atlantic City, NJ.

[7] Aluisio, IR Fontes, Lucas, CP Cavalcante\& Luiz, FQ Silveira 2012, 'Novel Automatic ModulationClassification using Correntropy Coefficient', XXX Brazilian Symposium on Telecommunication, Brasilia

[8] Ataollah Ebrahimzadeh \& Seyed Alireza Seyedin 2006, Proceedings of the 5th World Scientific and Engineering Academy and Society International Conference on Telecommunications and Informatics, May 27-29, 2006:Automatic Digital Modulation Identification in Dispersive Channels. World Scientific and Engineering Academyand Soc., Istanbul.

[9] Berezdivin, R, Breinig, R \&Topp, R 2002, 'Next-generation wireless communications concepts andtechnologies', IEEE Communications Magazine, vol. 40, no. 3, pp. 108.

[10] Dobre, OA, Abdi, A, Bar-Ness, Y \& Su, W 2007, 'Survey of Automatic Modulation Classification Techniques: Classical Approaches and New Trends', Communications, Institution of Engineering and Technology, vol. 1,no. 2, pp. 137-156.

[11] Fatima K Faek 2010, 'Digital Modulation Classification Using Wavelet Transform and Artificial NeuralNetwork', Journal of ZankoySulaimani, vol.1, no.13, pp. 59-70

[12] Ho, KC, Prokopiw, W \& Chan, YT 1995, 'Modulation Identification by the Wavelet Transform', Proceedings of the IEEE Military Communications Conference, California, vol. 2, pp. 886-890

[13] Jefferson, L Xu, Wei Su \&Mengchu Zhou 2011, 'Likelihood-Ratio Approaches to Automatic Modulation Classification', IEEE Transactions on Systems, Man, and Cybernetics - Part C: Applications and Reviews, vol. 41, no. 4, pp. 455-469

[14] Marina Petrova, Petri Mahonen\& Alfredo Osuna (eds) 2010, International Symposium on Wireless Communication System, September 19-22, 2010: Multi-Class Classification of Analog and Digital Signals in Cognitive Radios using Support Vector Machines. IEEE Soc., United Kingdom

[15] Maurizio A Bonuccelli, Francesca Lonetti\& Francesca Martelli (eds) 2006, Proceedings of the International Symposium on a World of Wireless, Mobile and Multimedia Networks, June 26-29, 2006: Tree Slotted Aloha: a New Protocol for Tag Identification in RFID Networks . IEEE Computer Soc., New York.

[16] Muhammad Waqar Aslam, Zhechen Zhu \&Asoke K Nandi (eds) 2010, Military Communications Conference, October 31-November 3, 2010 : Automatic Digital Modulation Classification Using Genetic Programming with K-Nearest Neighbor. IEEE Soc., San Jose, CA. 
[17] Ozaktas, HM, Arikan, O, Kutay, MA \&Bozdagt, G 1996,' Digital computation of the fractional Fourier transform', IEEE Transactions Signal Processing, vol. 44, no. 9, pp.2141-2150.

[18] Prakasam, P \&Madheswaran, M 2009, 'Reconfigurable Automatic Modulation Identification Hardware Module for Software Defined Radio Receivers', International Journal of Computer Theory and Engineering, vol. 1, no. 5, pp. 1793-8201.

[19] Sarker, JH, Hassan, M \&Halme, S 2002, 'Power level selection schemes to improve throughput and stability of slotted ALOHA under heavy load', Computer Communications, vol. 10, pp. 1719-1726.

[20] Xiao, H 2010, Proceedings of the Sarnoff Symposium, April 12-14, 2010: An Investigation of non-data-aided SNR Estimation techniques for Analog Modulation signals. IEEE Soc., New Jersey 